\title{
Patrones de resistencia antimicrobiana en uropatógenos gramnegativos aislados de pacientes ambulatorios y hospitalizados Cartagena, 2005-2008
}

\author{
Antimicrobial resistance pattern for gram-negative uropathogens \\ isolated from hospitalised patients and outpatients in Cartagena, \\ 2005-2008 \\ Raimundo Castro-Orozco ${ }^{1}$, Ana C. Barreto-Maya ${ }^{1}$, Heidy Guzmán-Álvarez², \\ Rolando J. Ortega-Quiroz ${ }^{1}$ y Lourdes Benítez-Peña ${ }^{1}$
}

1 Instituto de Investigaciones Biomédicas Alberto Montealegre (IDIBAM), Universidad de San Buenaventura, seccional Cartagena. Colombia. raimundo_castro_orozco@hotmail.com, anacarobm26@hotmail.com,rortega@usbctg.edu.co, lbenitez@usbctg.edu.co

2 Laboratorio de Atención a la Comunidad. Universidad de San Buenaventura, Seccional Cartagena. Colombia. heydicita02@ hotmail.com

Recibido 18 Mayo 2010/Enviado para Modificación 20 Diciembre 2010/Aceptado 28 Diciembre 2010

\section{RESUMEN}

Objetivo Determinar el patrón de resistencia antimicrobiana de bacterias patógenas asociadas a infecciones urinarias en pacientes ambulatorios y hospitalizados.

Métodos Se realizó un estudio descriptivo entre febrero de 2005 y noviembre de 2008 en el Laboratorio Clínico de la Universidad de San Buenaventura, Cartagena. La susceptibilidad antimicrobiana fue evaluada con el método de difusión en agar empleado la técnica de Kirby Bauer aplicando procedimientos normalizados.

Resultados Del total de muestras de orina analizadas (1384) durante el período de estudio, 32,9 \% (455) fueron urocultivos positivos, la mayoría de éstos (81,4\%) provenían de mujeres. Los agentes bacterianos más frecuentes fueron Escherichia. coli (60,1\%), Klebsiella pneumoniae (6,9 \%), Pseudomonas aeruginosa (6,6 \%), Proteus mirabilis (5,4\%) y Acinetobacter baumannii (1,4\%). Los aislamientos gramnegativos mostraron una alta resistencia a la ampicilina (84,3-100\%), amoxacilina/ácido clavulánico $(66,5-80,0 \%)$ y ciprofloxacina $(40,0-57,9 \%)$.

Conclusión Las bacterias gramnegativas fueron los principales agentes asociados a infecciones urinarias en esta población siendo $E$. coli el aislamiento más frecuente. La interpretación de los perfiles de susceptibilidad encontrados permite considerar como prudente la administración empírica de cefalosporinas de tercera generación como tratamiento inicial de las infecciones urinarias en esta población.

Palabras Clave: Bacteriuria, infecciones urinarias, pruebas de sensibilidad microbiana, farmacorresistencia microbiana (fuente: DeCS, BIREME). 


\section{ABSTRACT}

Objective Determining the microbial aetiology spectrum and antibiotic resistance pattern of uropathogens causing urinary tract infections in hospitalised patients and outpatients.

Methods A descriptive study was carried out between February 2005 and November 2008 at the San Buenaventura University's Clinical Laboratory in Cartagena. Antibiotic sensitivity was determined by the Kirby Bauer method.

Results Out of the total specimens $(1,384)$ analysed over the four-year study period, 455 of the urine samples $(32.9 \%)$ were culture positive, most $(81.4 \%)$ having come from females. The bacterium isolated most frequently was Escherichia coli $(60.1 \%)$ followed by Klebsiella pneumoniae (6.9\%), Pseudomonas aeruginosa (6.6\%), Proteus mirabilis (5.4\%) and Acinetobacter baumannii (1.4\%). The Gram-negative isolates displayed a high level of resistance to ampicillin (range 84.3-100\%), amoxicillin/clavulanic acid (range 66.5-80 \%) and ciprofloxacin (range 40-57.9\%). Conclusion Gram-negative bacteria were responsible for urinary tract infections in the patients involved in this study. The most commonly isolated bacteria were $E$. coli. Empirical administration of a third-generation cephalosporin for initial treatment of urinary tract infections in this population appears prudent from the perspective of antimicrobial susceptibility.

Key Words: Bacteriuria, urinary tract infection, microbial sensitivity test, drug resistance, microbe (source: $M e S H, N L M$ ).

$\mathrm{E}$ $\mathrm{n}$ el mundo, se consideran a las infecciones urinarias una de las principales causas de consulta y de hospitalización, reportándose aproximadamente 150 millones de casos al año, con diferencias en las frecuencias reportadas de acuerdo al grupo etario (1). En niños y niñas menores de un año, se reportan datos de $3,7 \%$ y $2 \%$, respectivamente; este patrón de frecuencia cambia en los infantes menores de 11 años (niñas, $3 \%$; niños, 1,1\%). En los adultos mayores, la prevalencia de bacteriuria asintomática oscila entre el $10 \%$ y el $50 \%$, frecuentemente asociada a bacteriemia $(1,2)$.

En cuanto al sexo, las infecciones urinarias entre mujeres y hombres se estima en 30:1, conforme se envejece esta razón tiende a igualarse, pero aún, con un ligero predominio del sexo femenino (3).

En cuanto al diagnóstico, se considera un hallazgo significativo todo recuento igual o superior a $10^{5}$ UFC de un mismo agente infeccioso, en un paciente con sintomatología clínica (1). Realizando este procedimiento de laboratorio se puede llegar a la identificación y cuantificación de los agentes patógenos presentes en la orina y, además, permite evaluar la sensibilidad a los antibióticos de primera y segunda elección, para así orientar la terapéutica. 
Actualmente, se reconoce la influencia que ejerce la emergencia de bacterias con patrones de resistencia en la gravedad de este tipo de infecciones, a tal punto que se considera un problema de salud pública, tanto en el ámbito nosocomial como en el comunitario $(5,6)$.

El conocimiento del mapa de sensibilidad antimicrobiana en cada zona geográfica permitiría hacer frente a esta situación problémica, ya que se podría mejorar el uso de antibióticos y se facilitaría la elección del tratamiento empírico.

Por todo lo anterior, el propósito de este estudio fue determinar los perfiles de resistencia antimicrobiana de bacterias gramnegativas asociadas a bacteriuria significativa, en muestras procesadas en el Laboratorio Clínico de la Universidad de San Buenaventura seccional Cartagena, durante el período comprendido entre Febrero de 2005 y Noviembre de 2008.

\section{MATERIALES Y MÉTODOS}

En este estudio de enfoque cuantitativo, con diseño epidemiológico transversal descriptivo, se estudiaron muestras de orina de pacientes ambulatorios y hospitalizados. El procesamiento de éstas se realizó según lo establecido en las normas y procedimientos para el diagnóstico microbiológico (7). Para la identificación de géneros y especies bacteriana, se utilizaron medios convencionales y sistemas de identificación $\mathrm{BBL}^{\mathrm{TM}} \mathrm{Cristal}^{\mathrm{TM}}$.

La susceptibilidad antimicrobiana fue evaluada con el método de difusión en agar empleado la técnica de Kirby-Bauer aplicando procedimientos normalizados (8). Los antibióticos utilizados fueron: trimetoprimsulfametoxazol-25 $\mu \mathrm{g}$ (SXT), amoxicilina/ácido clavulánico-20 $\mu \mathrm{g} / 10 \mu \mathrm{g}$ (AMC), ampicilina-sulbactam-20 $\mu \mathrm{g}$ (SAM), ciprofloxacina-5 $\mu \mathrm{g}$ (CIP), norfloxacina-10 $\mu \mathrm{g}$ (NOR), ceftazidima-30 $\mu \mathrm{g}$ (CAZ), ceftriaxona-30 $\mu \mathrm{g}$ (CRO), cefotaxima-30 $\mu \mathrm{g}(\mathrm{CTX})$, cefuroxima-30 $\mu \mathrm{g}(\mathrm{CXM})$, gentamicina-10 $\mu \mathrm{g}(\mathrm{GEN})$, amikacina-30 $\mu \mathrm{g}$ (AMK) y nitrofurantoina-300 $\mu \mathrm{g}$ (NIT).

Para establecer la relación entre las variables cualitativas se realizaron tablas de contingencia mediante la prueba $X^{2}$ y sólo cuando fue necesario se utilizó el test exacto de Fisher. 


\section{RESULTADOS}

Durante el período de estudio, se procesaron 1384 urocultivos, resultando 455 urocultivos positivos (pacientes ambulatorios: 318; pacientes hospitalizados: 137). De éstos se aislaron 423 bacterias gramnegativas asociadas a bacteriuria significativa, afectando mayoritariamente a individuos del sexo femenino $(82,3 \%, 348 / 423 ;$ IC95 \% 78,7-85,9; $\mathrm{p}=0,0001)$ y mayores de 15 años $(85,8 \%$, $363 / 423$; IC95 \% 82,5-89,1; $\mathrm{p}=0,0001)$.

El 89,6 \% (379/423) de estas cepas pertenecieron a la familia Enterobacteriaceae, presentándose un mayor número de aislamientos en los urocultivos de pacientes ambulatorios, en comparación a los provenientes de pacientes hospitalizados. Este comportamiento fue contrario al hallado en los aislamientos de bacterias gramnegativas no fermentadoras.

En este estudio, la gran mayoría de los urocultivos demostraron infección monobacteriana $(98 \%, 446 / 455)$ con predominio de Escherichia coli, tanto en pacientes ambulatorios $(69,9 \%, 209 / 299)$ como en pacientes hospitalizados $(36,3 \%, 45 / 124)$ (Tabla 1). En el resto de urocultivos positivos (32 casos) se identificaron bacterias grampositivas y/o levaduras.

Tabla 1. Distribución de bacterias gramnegativas uropatógenas según la procedencia de la muestra. 2005-2008

\begin{tabular}{lcc}
\hline \multirow{2}{*}{ Uropatógenos } & \multicolumn{3}{c}{ Pacientes } \\
& $\begin{array}{c}\text { Ambulatorios } \\
\mathrm{n}(\%)\end{array}$ & $\begin{array}{c}\text { Hospitalizados } \\
\mathrm{n}(\%)\end{array}$ \\
\hline Escherichia coli & $209(69,9)$ & $45(36,3)$ \\
Klebsiella pneumoniae & $16(5,4)$ & $13(10,5)$ \\
Pseudomonas aeruginosa & $8(2,7)$ & $20(16,1)$ \\
Proteus mirabilis & $19(6,4)$ & $4(3,2)$ \\
Acinetobacter baumannii & $0(0)$ & $6(4,8)$ \\
Otras enterobacterias & $45(15,0)$ & $28(22,6)$ \\
Otras bacterias gramnegativas & $2(0,6)$ & $8(6,5)$ \\
\hline Total & 299 & 124 \\
\hline
\end{tabular}

En cuanto a los perfiles de resistencia, 50-80\% de los aislamientos de $E$. coli, Klebsiella pneumoniae y Proteus mirabilis, presentaron resistencia frente a antibióticos betalactámicos con inhibidores de betalactamasas (ampicilina/sulbactam y amoxicilina/ácido clavulánico).

Los aislamientos de E. coli y K. pneumoniae presentaron altos porcentajes de resistencia frente a la cefalosporina de segunda generación cefuroxima $(63,0 \%$ y $61,5 \%$, respectivamente) y bajos porcentajes de resistencia (0- 
$36,4 \%$ ) frente a las cefalosporinas de tercera generación (ceftriaxona, cefotaxima y ceftazidima). A diferencia de éstas, $P$. mirabilis mostró bajos porcentajes de resistencia $(0-25,0 \%)$ frente a todas las cefalosporinas evaluadas. Además, todos estos aislamientos fueron resistentes a nitrofurantoina (Tabla 2).

Tabla 2. Perfiles fenotípicos de resistencia en enterobacterias uropatógenas 2005-2008

\begin{tabular}{lccc}
\hline Antibióticos & $\begin{array}{c}\text { E. coli } \\
\text { (IC95\%) }\end{array}$ & $\begin{array}{c}\text { K. pneumoniae } \\
\%(\text { IC95\%) }\end{array}$ & $\begin{array}{c}\text { P. mirabilis } \\
\%(\text { IC95\%) }\end{array}$ \\
\hline Penicilinas & $84,3(79,3-88,9)$ & $100(85,4-100)$ & NP \\
AMP & $64,6(58,1-71,4)$ & $57,1(42,5-71,7)$ & $57,1(42,5-71,7)$ \\
SAM & $66,5(60,1-72,9)$ & $80,0(65,4-94,6)$ & $50,0(35,4-64,6)$ \\
AMC & $63,0(56,5-69,6)$ & $61,5(46,9-76,1)$ & $10,0(0-24,6)$ \\
Cefalosporinas & $4,7(1,8-7,6)$ & $12,5(0-27,1)$ & 0 \\
CXM & $11,8(7,4-16,2)$ & $23,1(8,5-37,7)$ & 0 \\
CRO & $18,5(13,2-23,8)$ & $36,4(21,8-51,0)$ & $25,0(10,4-39,6)$ \\
CTX & & & \\
CAZ & $57,9(51,2-64,6)$ & $45,5(30,9-60,1)$ & $28,6(14,0-43,2)$ \\
Fluoroquinolonas & $60,2(53,6-66,8)$ & $18,8(4,2-33,4)$ & $18,2(3,6-32,8)$ \\
CIP & $11,8(7,4-16,2)$ & $16,7(2,1-31,3)$ & $9,1(0-23,7)$ \\
NOR & $53,1(46,3-59,9)$ & $22,2(7,6-36,8)$ & $33,3(18,7-47,9)$ \\
Aminoglucósidos & & & \\
AMK & & & \\
GEN & $66,1(59,7-72,5)$ & $43,5(28,9-58,1)$ & $43,8(29,2-58,4)$ \\
Otros & $7,1(3,6-10,6)$ & $60,9(46,3-75,5)$ & $100(85,4-100)$ \\
SXT & NIT &
\end{tabular}

AMP: ampicilia, SAM: ampicilina/sulbactam, AMC: amoxicilina/ácido clavulánico, CXM: cefuroxima, CRO: ceftriaxona, CTX: cefotaxima, CAZ: ceftazidima, CIP: ciprofloxacina, NOR: norfloxacina, AMK: amikacina, GEN: gentamicina, SXT: trimetoprim-sulfametoxazol, NIT: nitrofurantoina. NP: no probado.

Los porcentajes de resistencia en E. coli frente a los antibióticos: ampicilina, ceftazidima, gentamicina y trimetoprim-sulfametoxazol no presentaron diferencia estadísticamente significativa cuando se compararon según la procedencia del urocultivo. Para el resto de antibióticos evaluados, se encontró un número significativamente superior de bacterias resistentes, en los urocultivos de pacientes hospitalizados (Tabla 3).

Por otro lado, la multiresistencia de 3 hasta 5 antibióticos con mecanismo de acción diferentes fue demostrada en $57,8 \%$ (26/45) y 26,8 \% (68/209) de los urocultivos de pacientes hospitalizados y ambulatorios, respetivamente. Dentro de estas cepas multiresistentes, el perfil más frecuente fue SAMNOR-GEN-SXT. 
No se encontró diferencia estadísticamente significativa ( $p>0.05)$ en la variación anual de la resistencia encontrada en las cepas de $E$. coli frente a los antibióticos evaluados (Figura 1).

Por otra parte, Pseudomonas aeruginosa presento mayor susceptibilidad frente ceftazidima $(70,6 \%)$, piperacilina-tazobactam $(66,7 \%)$, cefepime $(66,7 \%)$, merepenem $(66,7 \%)$ y amikacina $(61,9 \%)$.

Tabla 3. Perfiles fenotípicos de resistencia en Escherichia coli, según la procedencia de las muestras (pacientes ambulatorios y hospitalizados)

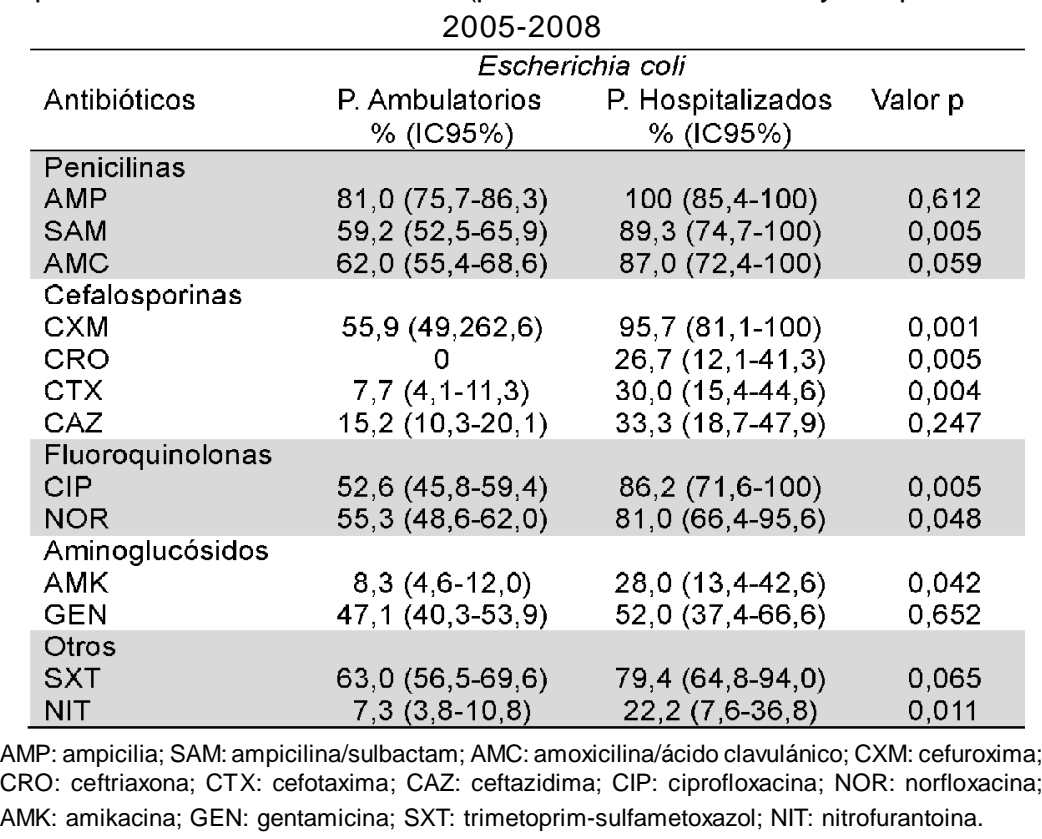

Todas las cepas de Acinetobacter baumannii fueron obtenidas en muestras de orina de pacientes hospitalizados. El $50 \%(3 / 6)$ de éstas presentaron resistencia a antibióticos de 3 o más familias diferentes. Para estas bacterias el patrón de multiresistencia más frecuente fue SAM-IPM-CIP-GEN. 
Figura 1. Tendencia del comportamiento de la resistencia antimicrobiana en cepas uropatógenas de E. coli aisladas de pacientes ambulatorios. 2005-2008

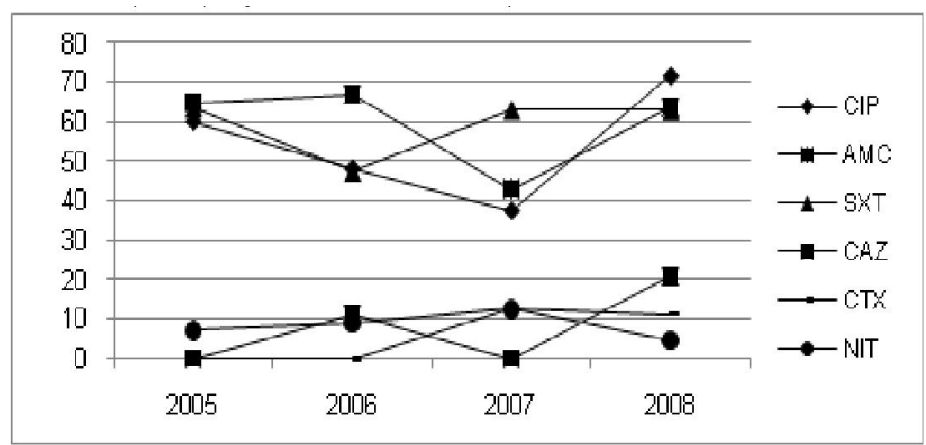

AMC: amoxacilina-ácido clavulánic; CAZ: ceftazidina; CIP: ciprofloxacina; CTX: cefotaxima; NIT: nitrofurantoina; SXT: trimetoprim-sulfametoxazol

\section{DISCUSIÓN}

Las infecciones urinarias se consideran una importante entidad clínica de alta morbilidad y causa frecuente de hospitalización en pacientes de todas las edades, los cuales pueden presentar desde una bacteriuria asintomática hasta cuadros más severos como la pielonefritis aguda con septicemia.

Tanto el diagnóstico como la terapéutica de estos procesos infecciosos deben tener una fundamentación microbiológica que permita orientar racionalmente el tratamiento empírico y, de esta forma, disminuir la creación, adaptación y diseminación de los mecanismos de resistencia bacteriana a los antibióticos de elección $(9,10)$.

Los resultados obtenidos en este estudio se relacionan con lo descrito por otros autores, en lo referente a la frecuencia de las bacterias uropatógenas y su distribución con respecto al sexo, a los grupos etarios afectados y a la procedencia del urocultivo $(3,11-21)$.

En cuanto a los porcentajes de resistencia antimicrobiana, las cepas de E. coli muestran valores similares a los reportados, excepto para ampicilinasulbactam y cefuroxima, los cuales presentaron menor actividad in vitro. En el caso de los aislamientos de K. pneumoniae y P. mirabilis, la resistencia encontrada para amoxicilina-ácido clavulánico es superior a la reportada en los referentes $(14,19-24)$. 
La implementación de una antibioticoterapia empírica está indicada en el tratamiento de las infecciones urinarias adquiridas en la comunidad. Dicho tratamiento varía de acuerdo a la edad del paciente, sexo, infecciones asociadas, agente infeccioso y el sitio anatómico de las vías urinarias (altas o bajas) comprometido. Esta conducta de acción es necesaria hasta obtener el resultado del urocultivo que permita tomar decisiones de acuerdo al patrón de sensibilidad del antibiograma. Para que un antibiótico pueda ser utilizado para tal fin, debe presentar una tasa de resistencia no superior al 10-20\% $(25,26)$.

Según lo anterior, los resultados microbiológicos de este estudio indican que los antibióticos que podrían ser utilizados empíricamente para el tratamiento de las infecciones urinarias en esta área geográfica serían nitrofurantoina, ceftriaxona, cefotaxima, ceftazidima y amikacina (Tabla 3).

Hay que resaltar que, si bien la tasa de resistencia de estos antibióticos se mantuvo inferior o igual al $20 \%$ durante los cuatro años de estudio, el antibiótico amikacina no podría ser considerado dentro de este grupo, ya que por su toxicidad no se le considera de elección en atención primaria. Su uso está indicado en pacientes con criterios de ingreso hospitalario $(1,27)$.

Ante la sospecha clínica de infección por Proteus sp., como evidencia de historia de nefrolitiasis cálcica por hiperoxaluria o infecciones urinarias recurrentes, entre otras $(1,7,11)$, los hallazgos obtenidos en este estudio señalan que los antibióticos con mayor actividad in vitro serían norfloxacina y las cefalosporinas de segunda y tercera generación, excepto ceftazidima.

Ninguno de los betalactámicos combinados con inhibidores de betalactamasas evaluados en este estudio presenta una tasa de resistencia que permita su uso en terapia de primera línea de las infecciones urinarias asociadas a enterobacterias. En relación con esto, se ha reportado que $E$. coli puede desarrollar resistencia a estas combinaciones por varios mecanismos, como por ejemplo la hiperproducción de betalactamasas tipo TEM-1 o AmpC, alteraciones en los canales de porinas y con menor frecuencia betalactamasas tipo TEM-1 mutantes $(14,28,29)$.

A pesar de que este estudio presenta limitaciones tales como: la característica cualitativa de la técnica utilizada para evaluar la susceptibilidad antimicrobiana y la posibilidad de sesgos de información y selección, la interpretación de los perfiles de resistencia encontrados permite considerar 
como prudente la administración empírica de cefalosporinas de tercera generación como tratamiento inicial de las infecciones urinarias en esta población

Agradecimientos: Los autores agradecen al personal que labora en el laboratorio clínico de atención a la comunidad de la Facultad de Ciencias de la Salud de la Universidad de San Buenaventura, seccional Cartagena.

\section{REFERENCIAS}

1. Echavarría J, Sarmiento E, Osores F. infección del tracto urinario y manejo de antibiótico. Acta Med Per 2006; 23(1):26-31.

2. Hooton TM. Recurrent urinary tract infection in women. Int J Antimicrob Agents 2001; 17:259-268.

3. Álvarez JC. Infecciones de vías urinarias en el Hospital Universitario del Norte. Salud Uninorte 2007; 23(1):9-18.

4. Díaz L, Cabrera LE, Fernández T, González O, Carrasco M, Bravo L. Etiología bacteriana de la infección urinaria y susceptibilidad antimicrobiana en cepas de Escherichia coli. Rev Cubana Pediatr. 2006; 78(3):2-9.

5. Spellberg B, Guidos R, Gilbert D. The epidemic of antibiotic-resistant infections: a call to action for the medical community from the Infectious Diseases Society of America. Clin Infect Dis. 2008; 46:155-64.

6. The cost of antibiotic resistance: effect of resistance among Staphylococcus aureus, Klebsiella pneumoniae, Acinetobacter baumannii, and Pseudomonas aeruginosa on length of hospital stay. Infect Control Hosp Epidemiol. 2002; 23:106-8.

7. Koneman EW, Allen SD, Dowell VR, Janda WN, Sommers HM, Winn WC. Diagnóstico microbiológico. $3^{\text {ra }}$ ed. Buenos Aires: Editorial Médica Panamericana; 1998.

8. National Committee for Clinical Laboratory Standards (NCCLS). Performance standards for antimicrobial susceptibility testing. M100-S11; 2000.

9. ECLM-European Urinalysis Group. European urinalysis guidelines. Scand J Clin Lab Invest. 2000; 60 (Supple. 231):1-7.

10. Graham JC, Galloway A. The laboratory diagnosis of urinary tract infections. J Clin Pathol. 2001; 54:911-919.

11. Velasco A. Infecciones de las vías urinarias. En: Paniagua EA. Manual de diagnóstico y terapéutica médica en atención primaria. 3ra edición. España. Ediciones Díaz de Santos S.A.; 2001. p. 833-842.

12. Robledo CG. Infección urinaria. En: Díaz FJ, Estrada S, Franco L, Jaramillo JM, Maestre AE, Ospina S, et al. editores. Fundamentos básicos de medicina, Microbiología de las infecciones humanas. 1를 Edición. Bogotá: Fondo editorial CIB; 2007. p. 826-831.

13. Sobel J, Kaye D. Urinary tract infections. En: MandellG, Bennet J, Dolin R editores. Principles and practice of infectious disease, 6th ed.Elsevier Inc; 2005. p. 875-905.

14. Kacmaz B. and Sultam N. In vitro susceptibilities of Escherichia coli and Klebsiella spp. to ampicillim-sulbactam and Amoxicillin-Clavulanic Acid. Jpn J Infect Dis 2007;60:227229.

15. Sweih N, Jamal W, Rotimi V. Spectrum and Antibiotic Resistance of Uropathogens Isolated from Hospital and Community Patients with Urinary Tract Infections in Two Large Hospitals in Kuwait. Med Princ Pract. 2005; 14:401-407.

16. Gales AC, Sader HS, Jones RN. The SENTRY Participants Group (Latin America) Urinary tract infection trends in Latin American hospitals: report from the SENTRY antimicrobialsurveillance program (1997-2000). Diagn Microbiol Infect Dis. 2002; 44:289-99. 
17. Hima-Lerible H, Menard D, Talarmin A. Antimicrobial resistance among uropathogens that cause community-adquired urinary tract infections in Bangui, Central African Republic. J Antimicrob Chemother. 2003; 51:192-4.

18. Ranjbar R, Haghi MT, Jonaidi N, Abedini M. The prevalence and antimicrobial susceptibility of bacterial uropathogens isolated from pediatric patients. Iranian J Public Health. 2009; 38(2):134-8.

19. Radrianirina F, Soares JL, Carod JF, Ratsima E, Thonnier V, Combe P, et al. Antimicrobial resistance among uropathogens that cause community-acquired urinary tract infections in Antananarivo, Madagascar. J Antimicrobial Chemother. 2007; 59:309-12.

20. García A, Hernández A, Salazar JL, Dávila J, Gómez LS. Etiología y resistencia antibiótica de las infecciones de vías urinarias adquiridas en la comunidad en Monterrey N.L. Rev Mex Urol. 2009; 69(2):45-8.

21. Gómez E, Plata M, Sejnaui J, Rico CL, Vanegas S. Resistencia de la E.coli en urocultivos de pacientes con sospecha de infección urinaria intra y extra-hospitalaria en la Fundación Santa Fe de Bogotá. Urol Colomb. 2009; 18(1):53-8.

22. Manges AR, Dietrich PS, Riley LW. Multidrug-resistant Escherichia coli clonal groups causing community-acquired pyelonephritis. Clin Infect Dis. 2004; 38:329-34.

23. Wassem R, Colodner R, Chazan B, Elias M, Raz R. Ten years surveillance of antimicrobial susceptibility of community-acquired Escherichia coli and other uropathogens in Northern Israel. IMAJ. 2007; 9:803-5.

24. Lujan DA, Pajuelo GR. Frecuencia y susceptibilidad antimicrobiana de patógenos aislados en infección del tracto urinario. Biomedica. 2008; 19:110-5.

25. Izquierdo R, Carranza R, Valenzuela JC, Fernández J. Etiología y resistencia bacteriana de la infección urinaria extrahospitalaria. Semergen. 1996; 25(1):11-4.

26. Speller DC. The clinical impact of antibiotic resistance. J Antimicrob Chemother. 1988; 22:583-6.

27. Mensa J. Guía terapéutica antimicrobiana. 17ª Edición. Barcelona (España): Elsevier Doyma S.L.; 2007.

28. Babic M, Hujer AA, Bonomo RA. What's new in antibiotic resistance? Focus on betalactamases. Drug Resist Updates. 2006; 9:142-156.

29. Stapleton P, Wu PJ, King A, Shannon K, French G, Phillips I. Incidence and mechanisms of resistance to the combination of amoxicillin and clavulanic acid in Escherichia coli. Antimicrob Agents Chemother. 1995; 39(11):2478-83. 